Article

\title{
Analyses and the Cause of Corrosion and Materials Loss at the Base of a Three-Phase Separator in Petroleum-Based Processing
}

\author{
H. Ding a,b* , H.B. Li a ${ }^{a}$, D.T. Qi ${ }^{a}$, Z. Zhang a, G.Q. Qi a,b, B. Wei a and X.H. Cai a
}

\begin{abstract}
a Tubular Goods Research Institute, China National Petroleum Corporation, State Key Laboratory for Performance and Structure Safety of Petroleum Tubular Goods and Equipment Materials, Shaanxi Xi' an 710065, China;

b. Department of Applied Chemistry, School of Science, Northwestern Polytechnical University, Xi'an 710072, China;
\end{abstract}

\begin{abstract}
The three-phase separator was perforated after four years' service. The perforation position is at the bottom of the vessel under the baffle plate in an oil transfer station. In order to find out the causes of corrosion and perforation of the vessel, it was investigated by chemical analysis using a direct-reading spectrometer, microstructure examined by optical microscope(OM), and lab simulated corrosion experiments by electrochemical workstation, and the corrosion products examined with scanning electron microscopy(SEM), energy dispersive spectrometer(EDS) in this paper. The analyses indicated that the coating at the base of the pressure vessel failed and this led to the direct exposure of the carbon steel plate to the corrosive medium. The reason for the corrosion and perforation is that base material in the concealed place of the vessel contacts with the medium directly and corrodes due to coating failure. If the vessel carbon steel connects with the baffle plate stainless steel to form a hetero-metallic couple in the corrosive medium, the vessel inner wall's corrosion will be further accelerated.
\end{abstract}

Keywords: Three-phase separator; Perforation; Corrosion; Galvanic;

\section{Introduction}

Oil is a kind of non-renewable resources, it exploited out from the deep stratum of earth will inevitably carries out some formation water and associated gas. Three-phase separator is used for separating the produced wellhead fluids into gas, oil and water by their density difference. Because of the existence of $\mathrm{CO}_{2}$ and $\mathrm{H}_{2} \mathrm{~S}$ acidic gas media in the gaseous products, highly mineralized produced water, especially the chloride ion. After four years' service, a three-phase separator's bottom was perforated. A $30 \times 50 \mathrm{~mm}$ perforation was found in the bottom plate, and the thickness of the plate near the perforation position is seriously reduced, as shown in Fig. 1. There are two three-phase separators in the oil transfer station. The crude oil separated from the separators is heated by the heating furnace and then pumped to the combined station. The oil transfer station is the main block of the joint station, producing $900 \mathrm{t} / \mathrm{d}$ of oil. The produced water is about $300 \mathrm{~m}^{3} / \mathrm{d}$ and is injected back in stratum. 


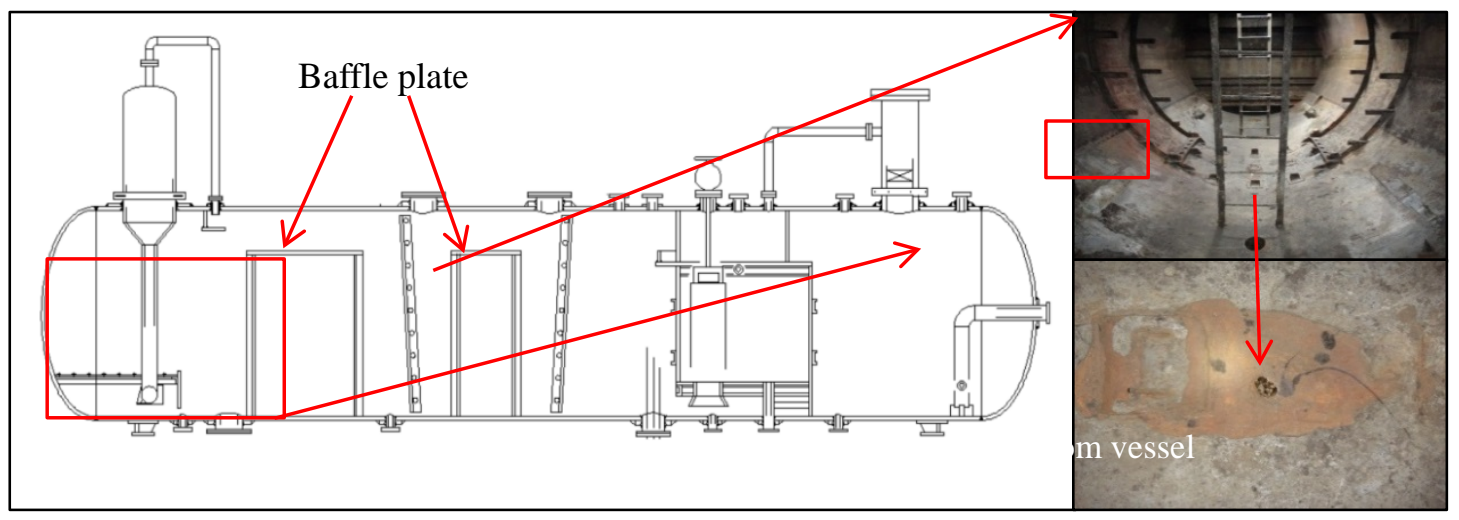

Figure 1. The perforating position of three-phase separator.

The plate of the vessel is made of Q245R carbon steel with a thickness of $12 \mathrm{~mm}$. Samples were taken from the etched and perforated bottom plate of the vessel as shown in Fig. 2(a), marked as hole A. The baffle plates made by 316L stainless steel with a thickness of $0.5 \mathrm{~mm}$ as shown in Fig. 2(b).
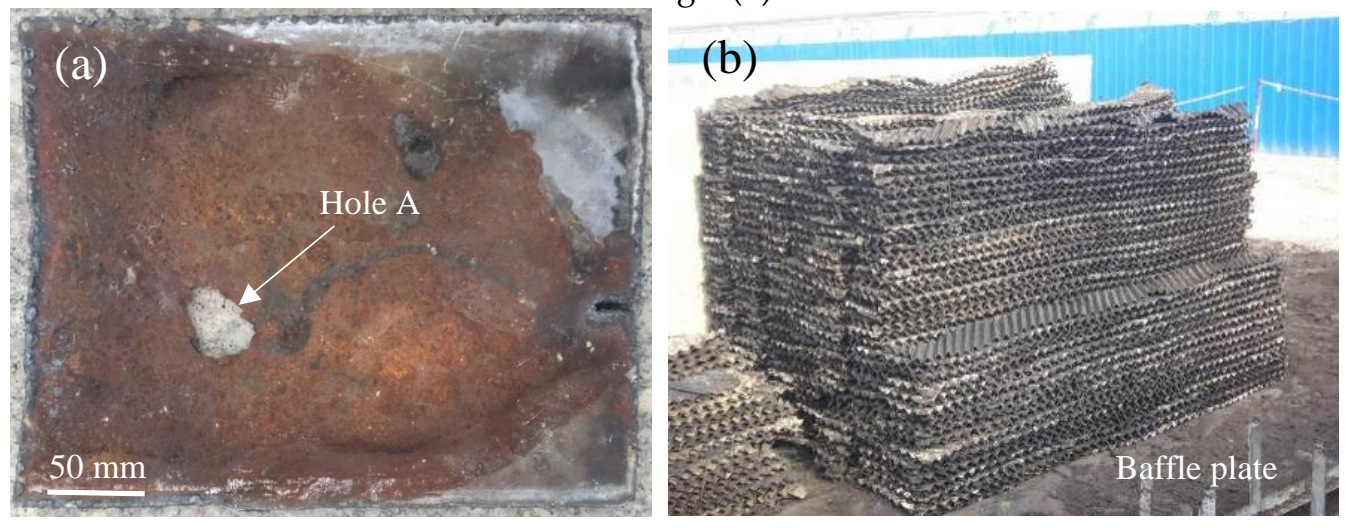

Figure 2. Failure samples of three-phase separator. (a) The perforated bottom plate of the vessel; (b) The 316L stainless steel baffle plates.

Metal made equipment were corroded by hydrogen sulfide has been investigated in some of the literatures[1-3], other researchers also studied the vessels and pipe chlorideinduced corrosion[4] and stress assisted corrosion[5-7]. Although, many scholars studied the three-phase separator were about the designation through computational fluid dynamics[8-11], simulate the internal flow field of the three-phase separator[12] and detection technology[13]. However, the corrosion of a three-phase separator is rarely been studied. In this paper, we studied a three-phase separator perforation in the bottom of the vessel, which corroded by the medium environment containing hydrogen sulfide, carbon dioxide, oxygen, and chloride ions [14-17].

The objective of this work is to find out the causes of corrosion and perforation of the vessel, the physical and chemical properties and corrosion resistance of the samples were tested comprehensively. According to the test results, combined with the field service conditions for comprehensive analysis, finally find out the cause of failure and put forward suggestions.

\section{Experimental}

In order to find out the corrosion cause of the three-phase separator, a series of experiments were carried out. According to GB/T 4336-2016 standard[18], the chemical composition samples were taken from the perforated vessel bottom plate was analysis by ARL4460 photoelectric direct reading spectrometer. The perforated vessel bottom plate 
sample was cut and prepared for the metallurgical examination. The MeEF4M metallographic microscope and image analysis system were used to observe the microstructure and grain size of the perforated vessel bottom plate.

To study the corrosion mechanism of the sample in service-environment, the hightemperature high-pressure autoclave was utilized to simulate the corrosion behavior of the of failure vessel samples in the laboratory[19] as shown in Fig. 3. The specific ion concentration is shown in Table 1. The simulated solution was prepared by using deionized water and pure grade chemical reagents $\left(\mathrm{Na}_{2} \mathrm{SO}_{4}, \mathrm{NaHCO}_{3}, \mathrm{NaCl}, \mathrm{CaCl}_{2}, \mathrm{KCl}, \mathrm{MgCl}\right.$, and $\mathrm{SrCl}_{2}$ ) to prepare the simulated solution. The $\mathrm{pH}$ value of the simulated solution was fitted by using dilute hydrochloric acid or sodium hydroxide solution. The exact simulation test parameters are given in Table 2.

Table 1. Simulated ionic composition of aqueous solution.

\begin{tabular}{ccccccccc}
\hline Ion $(\mathbf{m g} / \mathrm{L})$ & $\mathbf{H C O}_{3}{ }^{-}$ & $\mathbf{C l}^{-}$ & $\mathbf{S O}^{2-}$ & $\mathbf{C a}^{2+}$ & $\mathbf{M g}^{2+}$ & $\mathbf{S r}^{2+}$ & $\mathbf{K}^{+}$ & $\mathbf{N a}^{+}$ \\
\hline Concentration & 110 & 70700 & 332.7 & 7519 & 770.5 & 406.5 & 558.4 & 32800 \\
\hline
\end{tabular}

Table 2. Simulated corrosion test conditions.

\begin{tabular}{cccccccc}
\hline \multirow{2}{*}{ Temperature } & $\begin{array}{c}\text { Total } \\
\text { Pressure }\end{array}$ & \multicolumn{2}{c}{ Partial pressure of Partial pressure } & Medium & Time & Oxygen & $\begin{array}{c}\text { Flow } \\
\text { velocity }\end{array}$ \\
\hline $40^{\circ} \mathrm{C}$ & $0.5 \mathrm{MPa}$ & $0.03 \mathrm{MPa}$ & $0.01 \mathrm{MPa}$ & Simulated solution & $168 \mathrm{~h}$ & Contain & 0 \\
\hline
\end{tabular}

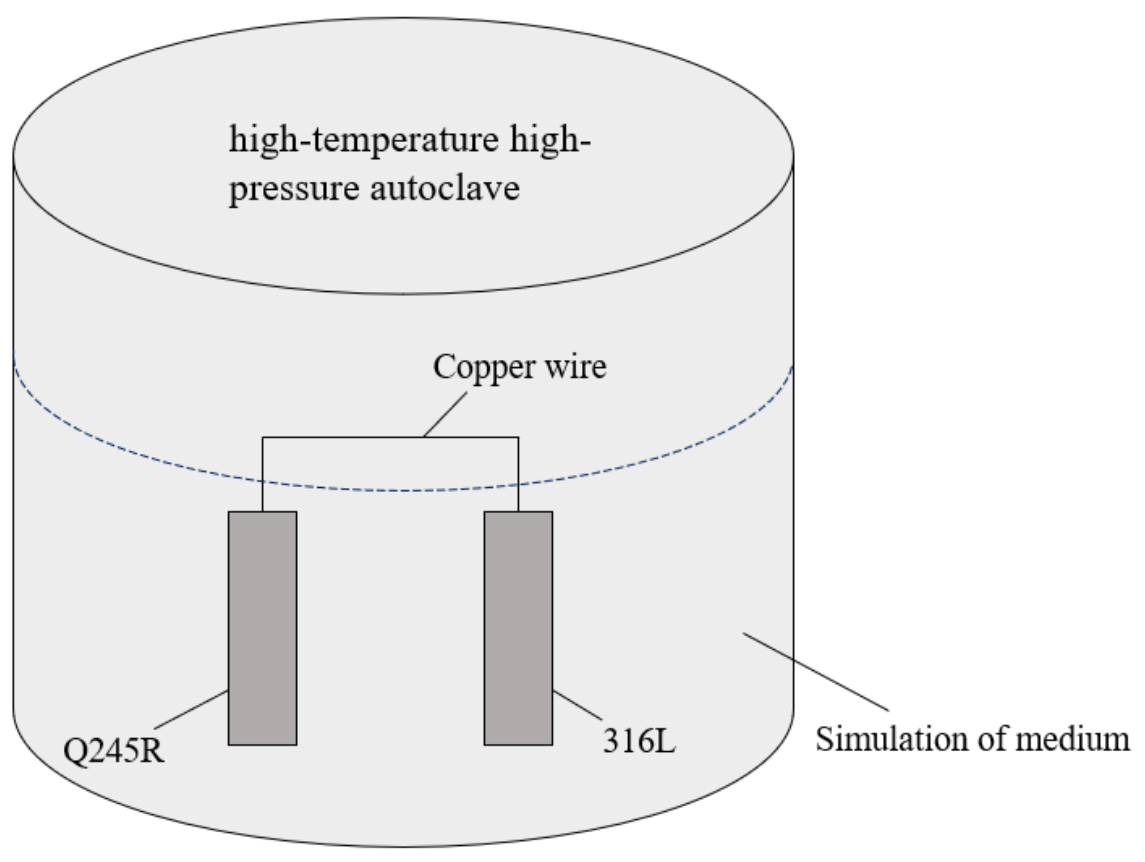

Figure 3. The metallographic structure of the perforated sample base material.

Corrosion simulation test samples were taken from the vessel bottom plate, with sampling specifications of $50 \mathrm{~mm} \times 10 \mathrm{~mm} \times 3 \mathrm{~mm}$. As the baffle plate is relatively thin, the hanging specimens were taken from the baffle plate with a length of $50 \mathrm{~mm}$ and a width of $10 \mathrm{~mm}$, so the two kind of samples' area are almost the same. Use tin-welded and copper wire to connect the different material hanging specimen, and the welding end is sealed with silica gel.

The electrochemical workstation was used to test the open-circuit potential (OCP) of the Q245R carbon steel sample, 316L stainless steel sample, and Q245R carbon steel and 316 stainless steel coupling sample were tested at $40^{\circ} \mathrm{C}$ and saturated carbon dioxide 
respectively. Besides, the dynamic potential polarization curves of Q245R carbon steel sample and 316L stainless steel sample were tested either. The potential scanning parameters of OCP are given in Table 3.

Table 3. Electrochemical test parameters of OCP.

\begin{tabular}{ccc}
\hline Sample & Scan range $(\mathbf{m V})$ & Scan rate $(\mathrm{mV} / \mathbf{s})$ \\
\hline Carbon steel & ocp-250 ocp +500 & 0.5 \\
\hline Stainless steel & ocp-250 ocp +1500 & 0.5 \\
\hline
\end{tabular}

Finally, the samples near the perforation site of the bottom plate were analyzed by TESCAN VEGA3 XMU scanning electron microscope (SEM) energy dispersive spectrometer (EDS).

\section{Results and discussion}

\subsection{Chemical composition}

The material composition of the sample is shown in Table 4. As the result, the chemical composition of the perforated sample conforms to the requirements of the GB 7132014[20] Q245R carbon steel, this standard identical to ISO 9328-2.

Table 4. Chemical composition of the perforated sample (wt.\%).

\begin{tabular}{cccccccccccccc}
\hline Element & $\mathbf{C}$ & $\mathbf{S i}$ & $\mathbf{M n}$ & $\mathbf{P}$ & $\mathbf{S}$ & $\mathbf{C r}$ & $\mathbf{M o}$ & $\mathbf{N i}$ & $\mathbf{N b}$ & $\mathbf{V}$ & $\mathbf{T i}$ & $\mathbf{C u}$ & $\mathbf{A l}$ \\
\hline GB 713-2014 & $\leq 0.20$ & $\leq 0.35$ & $0.50 \sim 0.10$ & $\leq 0.03$ & $\leq 0.01$ & $\leq 0.03$ & $\leq 0.03$ & $\leq 0.08$ & $\leq 0.05$ & $\leq 0.05$ & $\leq 0.03$ & $\leq 0.03$ & $\geq 0.02$ \\
Measured & 0.13 & 0.21 & 0.53 & 0.015 & $<0.008$ & $<0.1$ & $<0.03$ & 0.011 & $<0.02$ & $<0.1$ & $<0.015$ & $<0.02$ & 0.03 \\
\hline
\end{tabular}

\subsection{Metallographic analysis}

The metallurgical structure of the perforated vessel bottom plate sample is Ferrite+Pearlite $(\mathrm{F}+\mathrm{P})$ with a grain size of 8.0 and no abnormal structure was found around the corrosion perforation area, as shown in Fig. 4. The grain size is a kind of characterization of the metallurgical, the larger the grain size, the smaller the crystal shape, the better the strength, toughness and plasticity.
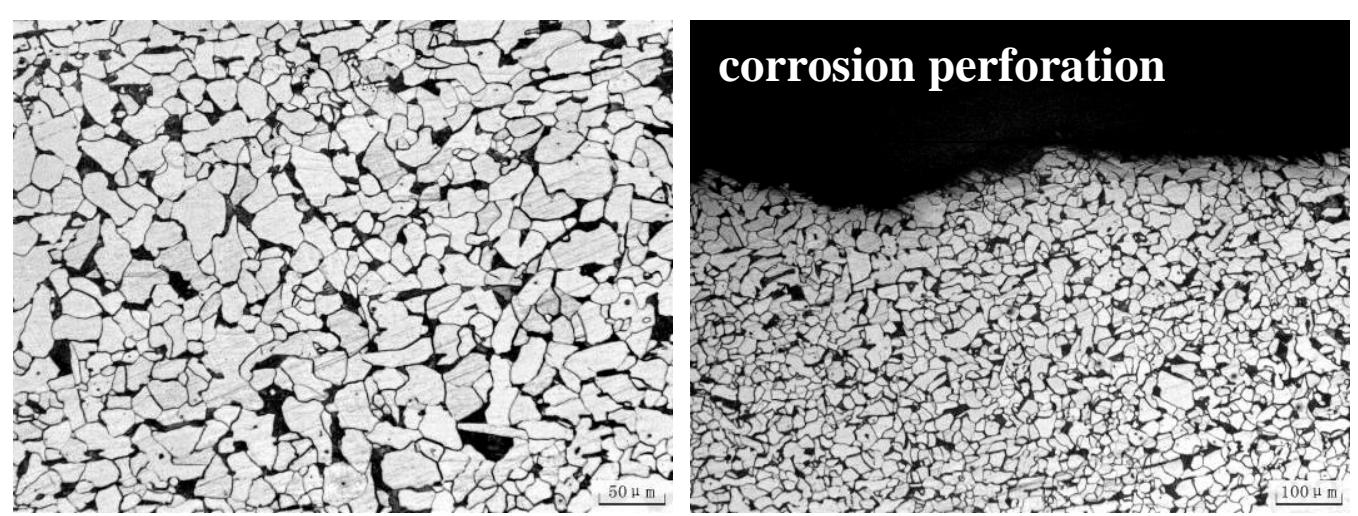

Figure 4. (a) Metallographic structure of the base material; (b) Metallographic structure around the corrosion perforation.

\subsection{Simulated corrosion analysis}

The corrosion rate results are shown in Table 5. The average corrosion rate of carbon steel is $0.5347 \mathrm{~mm} / \mathrm{a}$ which connected with stainless steel, and that of carbon steel samples without connection with stainless steel is $0.3744 \mathrm{~mm} / \mathrm{a}$. The macroscopic morphology of the specimens before film removal is shown in Fig. 5, and the macroscopic morphology 
after film removal is shown in Fig. 6. According to the corrosion degree specified in NACE RP-0775-2005[21], the test results are classified as severe corrosion $(>0.25 \mathrm{~mm} / \mathrm{a})$.

Table 5. Corrosion rate result of the specimens.

\begin{tabular}{ccc}
\hline Condition & Corrosion rate $(\mathbf{m m} / \mathbf{a})$ & $\begin{array}{c}\text { Average corrosion rate } \\
(\mathbf{m m} / \mathbf{a})\end{array}$ \\
\hline \multirow{3}{*}{ Carbon steel + Stainless steel } & 0.53 & \\
& 0.59 & 0.53 \\
\hline \multirow{2}{*}{ Carbon steel } & 0.49 & \\
& 0.35 & 0.37 \\
\hline
\end{tabular}

(a)

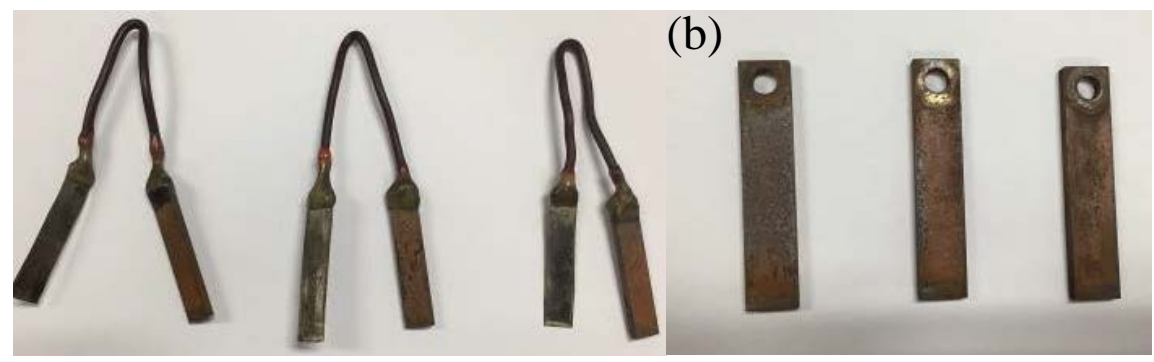

Figure 5. The macroscopic morphology of the sample before film removal (a) Carbon steel connected to the stainless steel; (b) Carbon steel.

(a)

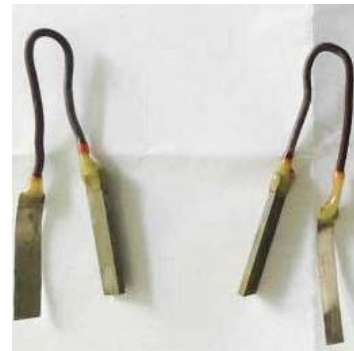

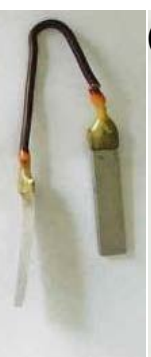

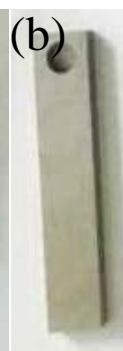

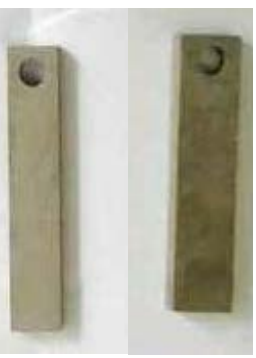

Figure 6. The macroscopic morphology of the sample after film removal (a) Carbon steel connected to the stainless steel; (b) Carbon steel.

\subsection{Electrochemical experimental analysis}

The OCP curve of different materials over time is shown in Fig. 7. It can be observed that the OCP of Q245R carbon steel alone is about $-0.717 \mathrm{~V}$ vs $\mathrm{AgCl} / \mathrm{Ag}$, and that of $316 \mathrm{~L}$ stainless steel alone is about $-0.45 \mathrm{~V}$ vs $\mathrm{AgCl} / \mathrm{Ag}$. The coupling potential (CP) of Q245R carbon steel and 316L stainless steel is $-0.706 \mathrm{~V}$ vs $\mathrm{AgCl} / \mathrm{Ag}$.

Fig. 8 shows the dynamic potential polarization curves of Q245R carbon steel and 316 L stainless steel. The corrosion of Q245R carbon steel is active dissolution, and the corrosion potential is $-0.7661 \mathrm{~V}$ vs AgCl$/ \mathrm{Ag}$, the corrosion current is $1.48 \times 10^{-4} \mathrm{~A} / \mathrm{cm}^{2}$. The current of anodic polarization curve increases rapidly with a positive shift of potential. The $316 \mathrm{~L}$ stainless steel is passivated, and the passivation current density is about $5 \times 10^{-6}$ $\mathrm{A} / \mathrm{cm}^{2}$, the pitting potential is about $0.1 \mathrm{~V}$ vs AgCl/Ag. 


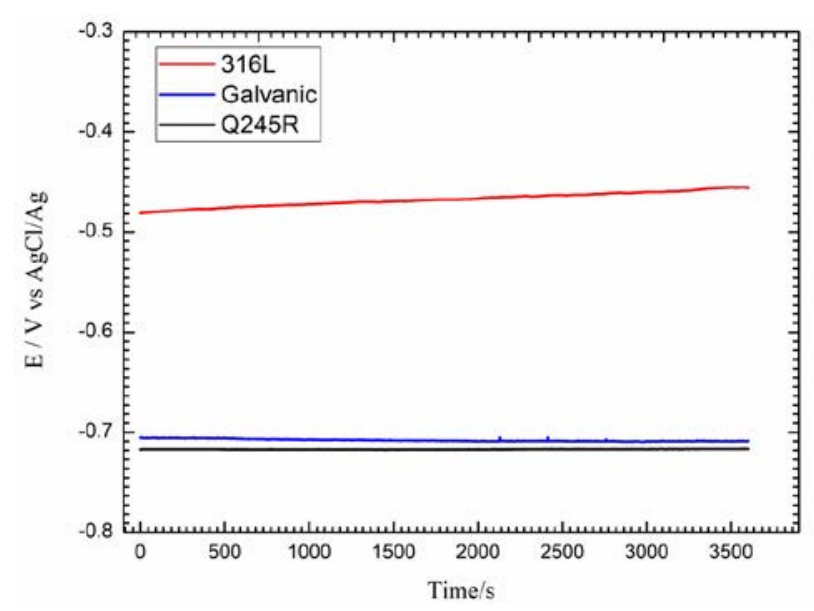

Figure 7. Curves of OCP of different materials with time.

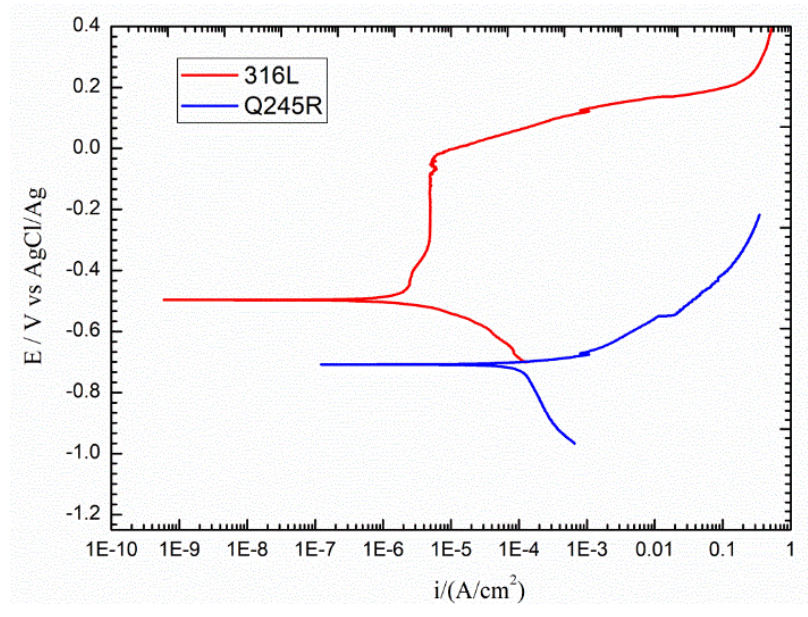

Figure 8. The dynamic potential polarization curve in the simulated liquid.

The OCP of Q245R carbon steel is negative $240 \mathrm{mV}$ compared with that of 316L stainless steel as shown in Fig. 7, and the corrosion potential of Q245R carbon steel is also significantly lower than that of 316L stainless steel as shown in Fig. 7. Therefore, when they are coupled, the Q245R carbon steel is the anode, the positive potential shift, the anode reaction rate increases, the cathode reaction is inhibited. 316L stainless steel is the cathode, the negative potential shift, the cathode reaction rate increases, the anode reaction is inhibited. On the other hand, the corrosion current of Q245R carbon steel is $1.48 \times 10^{-4} \mathrm{~A} / \mathrm{cm}^{2}$, much greater than the corrosion current of 316 stainless steel. Therefore, the coupling potential of Q245R carbon steel and 316L stainless steel is only slightly positive than that of Q245R carbon steel. However, according to the polarization curve, the current of Q245R carbon steel increases rapidly with the positive shift of the potential, so the coupling current will greatly increase, the anode of Q245R carbon steel is significantly promoted, that is, the corrosion accelerates.

\subsection{Electron microscope analysis}

The SEM image of the sample near the perforated hole A in Fig. 2 as shown in Fig. 9(a). The surface is relatively concave and convex with a lot of pitting pits. The pits local magnification is showed in Fig. 9(b), indicating that corrosion products are loose. 

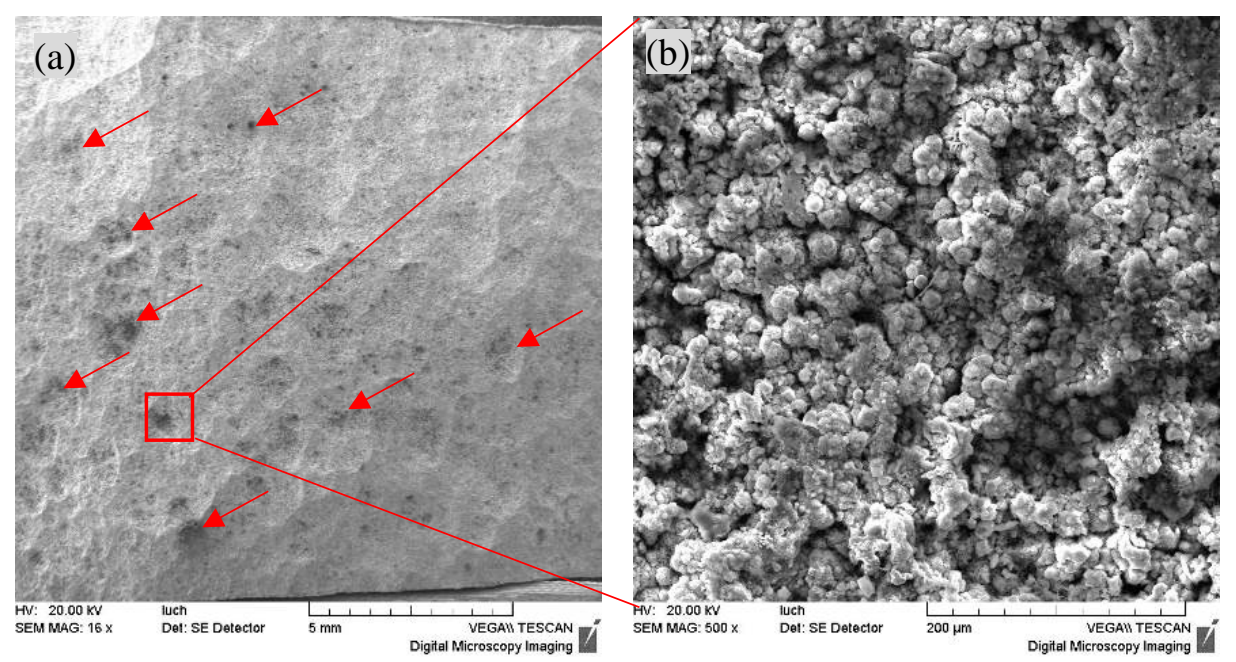

Figure 9. (a) SEM image near the perforated hole A; (b) Corrosion products in the pitting pits.

Other micro corrosion perforations were found besides the hole A in Fig. 2, one of those SEM image as shown in Fig. 10(a), the micro corrosion perforation is elliptic, with a diameter of $1.7 \mathrm{~mm}$. The microstructure of corrosion products near the micro corrosion perforation as shown in Fig. 10(b), and the corrosion products are abundant.
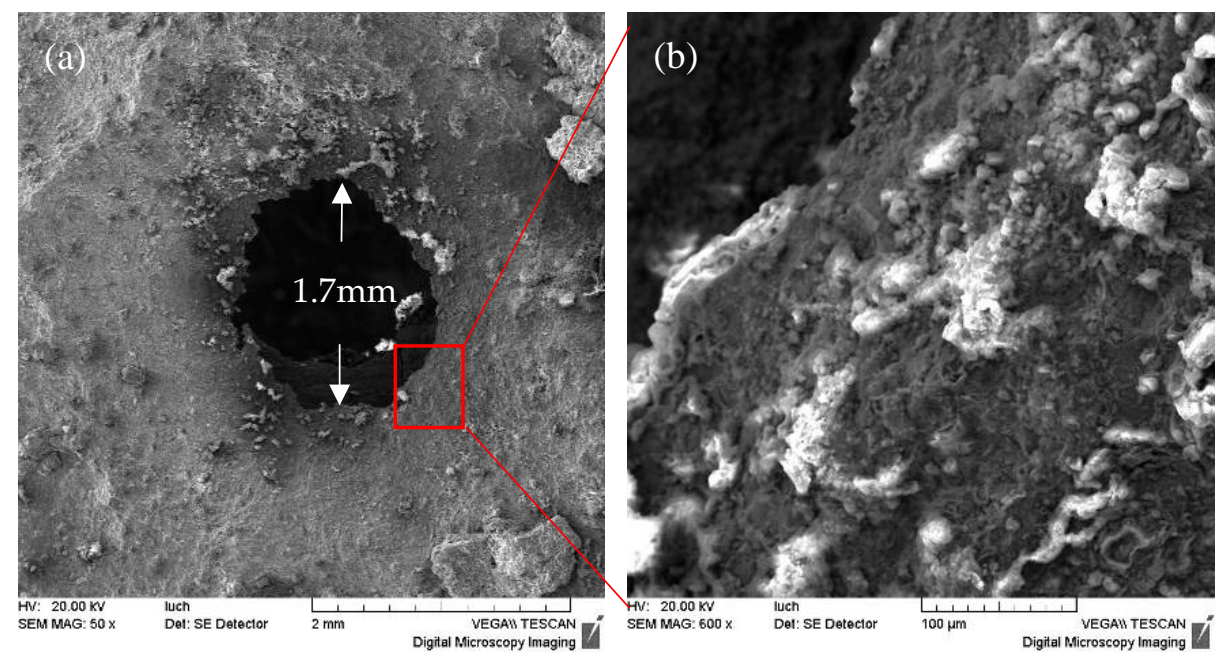

Figure 10. (a) SEM image of the micro corrosion perforation; (b) The microstructure of corrosion products near the micro corrosion perforation.

EDS was performed to analyze the distal and proximal ends of the micro corrosion perforation in Fig. 10(a). The specific inspection locations are showed in Fig. 11, and the detection results are presented in Table 6. According to the test results, the $\mathrm{Cl}$ and S element content of Spectrum 2 were higher than Spectrum 1, indicating that the chloride ion and Sulfur plays an important role in the formation of corrosion perforation. 


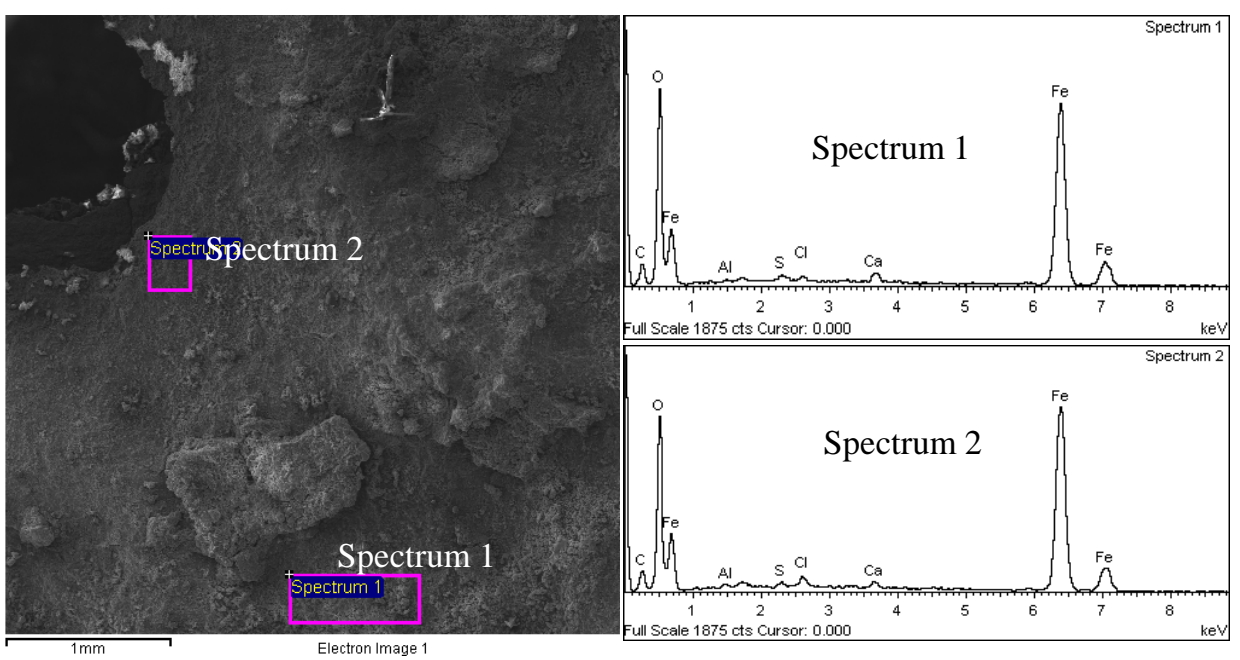

Figure 11. EDS analysis of the corrosion perforation.

Table 6. Energy dispersive spectrometer (EDS) analysis of corrosion perforation result (wt.\%).

\begin{tabular}{cccccccccc}
\hline Element & C & O & Al & S & Cl & Ca & Fe & Tb & Total \\
\hline Spectrum 1 & 8.72 & 32.00 & $/$ & $/$ & 0.41 & 1.06 & 54.66 & 3.16 & 100.00 \\
Spectrum 2 & 9.16 & 29.80 & 0.41 & 0.45 & 0.81 & 0.61 & 58.76 & $/$ & 100.00 \\
\hline
\end{tabular}

\section{Root cause analysis}

Based on the above test results and analysis, the chemical composition of the samples submitted for inspection meets the requirements of the GB 713-2014 standard for Q245R carbon steel. The metallographic structure of the perforation site is Ferrite+Pearlite $(\mathrm{F}+\mathrm{P})$, and there is no abnormality in the structure around the perforation, which the materials of the samples submitted for inspection meet the standard requirements.

The simulated corrosion test shows that the corrosion rate of single Q245R carbon steel is $0.3744 \mathrm{~mm} / \mathrm{a}$ as shown in Table 4 , which is serious corrosion $(>0.25 \mathrm{~mm} / \mathrm{a})$. When the sacrificial anode is consumed and the bottom plate of the vessel is not coupled with the corrugated plate, if the inner wall of the vessel is in direct contact with the medium, the corrosion of the inner wall of the vessel will be reduced at a rate of about $0.37 \mathrm{~mm} / \mathrm{a}$. When Q245R carbon steel contact with 316L stainless steel, because the corrosion potential difference between them is $240 \mathrm{mv}$, forming a pair of heterogeneous metal points, that Q245R carbon steel is the anode of potential pair. Its potential shifts forward as shown in Fig. 7 and Fig. 8. The corrosion current increases sharply and the corrosion accelerates obviously. Similarly, the simulation test also shows that the corrosion rate of Q245R carbon steel after coupling is $0.5347 \mathrm{~mm} / \mathrm{a}$ as shown in Table 4, which is significantly higher than that of single Q245R carbon steel, thus the couple pair further promotes the corrosion of Q245R carbon steel.

The SEM analysis of the failed vessel showed that near the perforation site surface is relatively concave and convex with a lot of pitting pits as shown in Fig. 9(a, and there were abundant loose corrosion products on the surface as shown in Fig. 9(b), which indicated that serious pitting corrosion occurred in and around the perforation site of the vessel. Other micro corrosion perforations were found besides the hole A in Fig. 2, one of those SEM image as shown in Fig. 10(a), the micro corrosion perforation is elliptic, with a diameter of $1.7 \mathrm{~mm}$. EDS analysis result of the hole as shown in Fig. 11, the closer to the micro corrosion perforation, the higher the chloride and sulfur content. Therefore, indicating that the chloride ion and sulfur plays a key role in the formation of pitting pits, eventually leading to corrosion perforation. 
During the four years of service, the sacrificial anode of the three-phase separator was replaced twice in the process of maintenance, and the perforation occurred at the bottom of the vessel under the baffle plate, which is a hidden place and not easy to find. The enormous consumption of sacrificial anode indicates that the coating has fallen off or the inner wall of the vessel has been directly contacted with the medium. When the sacrificial anode is exhausted, the vessel inner wall which protected by the sacrificial anode will be failed. Combining with the above test results, it is shown that serious corrosion will occur on the inner plate of the vessel when the substrate contacts with the medium directly, the Q245R carbon steel and baffle plate 316L stainless steel coupled form galvanic corrosion[22-24], the corrosion will accelerate significantly.

\section{Conclusions and recommendations}

The metallographic structure of the base material of the vessel sample is normal, and the chemical composition meets the quality requirements of Q245R carbon steel in GB 7132014 standard. The reason for the corrosion perforation of the base material of the container sample is that the metal base material in the hidden part of the container is directly in contact with the medium, mainly oxygen corrosion, and a small amount of carbon dioxide corrosion and hydrogen sulfide corrosion. The inner wall of the container sample submitted for inspection can form serious corrosion when the substrate is in direct contact with the medium under working conditions. If it is in contact with corrugated plate stainless steel to form a heterometallic couple, the corrosion will be further accelerated.

It is suggested to strengthen the inspection of corrosion risk in hidden parts of containers in the process of reconditioning, without leaving dead corners, especially for vessels with obvious consumption of sacrificial anodes. Perfect insulation measures between the baffle plate and support to prevent direct contact of dissimilar metals and galvanic corrosion.

Acknowledgement: This work has been supported by Tubular Goods Research Institute, China National Petroleum Corporation, State Key Laboratory for Performance and Structure Safety of Petroleum Tubular Goods and Equipment Materials.

\section{References}

1. Sung Jin Kim, Jin Ho Park, Kyoo Young Kim. Effect of microstructure on sulfide scale formation and corrosion behavior of pressure vessel steel in sour environment. Materials Characterization 111 (2016) 14-20.

2. Shugen $\mathrm{Xu}$, Shengjun Huang, Deguo Guo, et al. Failure analysis of a carbon steel pipeline exposed to wet hydrogen sulfide environment. Engineering Failure Analysis 71 (2017) 1-10.

3. G. Domizzi, G. Anteri, J. Ovejero-Garcia. Infuence of sulphur content and inclusion distribution on the hydrogen induced blister cracking in pressure vessel and pipeline steels. Corrosion Science 43 (2001) 325-339.

4. Chengtao Wang, Wei Li, Yuqiao Wang, et al. Chloride-induced stray current corrosion of Q235A steel and prediction model. Construction and Building Materials 219 (2019) 164-175.

5. K. Ravindranath, B. Al-Wakaa, N. Tanoli, et al. Stress assisted corrosion in a crude oil production tube made from L80 steel. Engineering Failure Analysis, 74 (2017) 142-149.

6. J. Orlikowski, S. Krakowiak. Pitting corrosion and stress-corrosion cracking of buffer tanks in a brewery. Engineering Failure Analysis 29 (2013) 75-82.

7. Tangqing $\mathrm{Wu}$, Maocheng Yan, Libao Yu, et al. Stress corrosion of pipeline steel under disbonded coating in a SRB-containing environment. Corrosion Science 157 (2019) 518-530.

8. Mehdi Mostafaiyan, Mohammad Reza Saeb, Alireza Emami Alorizi, et al. Application of evolutionary computational approach in design of horizontal three-phase gravity separators. Journal of Petroleum Science and Engineering Volume 119, July 2014, Pages 28-35.

9. Thuy Thi Le, Son Ich Ngo, Young-Il Lima, et al. Effect of simultaneous three-angular motion on the performance of an air-wateroil separator under offshore operation. Ocean Engineering 171 (2019) 469-484.

10. Thuy Thi Le, Son Ich Ngo, Young-Il Lima, et al. Three-phase Eulerian computational fluid dynamics of air-water-oil separator under off-shore operation. Journal of Petroleum Science and Engineering Volume 171, December 2018, Pages 731-747.

11. Ahmadreza Ghaffarkhah, Mohammadjavad Ameri Shahrabi, Mostafa Keshavarz Moraveji , et al. Application of CFD for designing conventional three phase oilfield separator. Egyptian Journal of Petroleum (2017) 26, 413-420. 
12. Ping Yu, Shilei Liu, Yaohua Wang, et al. Study on Internal Flow Field of the Three-phase Separator with Different Entrance Components Procedia Engineering 31 (2012) 145-149.

13. Marius Vileiniskis, Rasa Remenyte-Prescott, Dovile Rama, et al. Fault detection and diagnostics of a three-phase separator. Journal of Loss Prevention in the Process Industries Volume 41, May 2016, Pages 215-230.

14. Jing-Lu Fei, Xiao-Lei Yang, Sheng-Hui Wang, et al. Root causes analysis and countermeasures on unexpected perforation of tee pipe in the steam system of specialty chemical plant. Engineering Failure Analysis 104 (2019) 796-806.

15. Z.D. Fan, J.S. Du, Z.B. Zhang, et al. Internal leakage of plate heat exchangers caused by cooperation of pitting, crevice corrosion, and fretting. Engineering Failure Analysis 96 (2019) 340-347.

16. Liu Yucheng, Zhang Yinlong, Yuan Jianmei, et al. Research on corrosion perforation on pipeline by media of high salinity acidic oil-water mixture. Engineering Failure Analysis 34 (2013) 35-40.

17. Robert E. Melchers a, Robert J. Jeffrey a, Kayley M. Usher. Localized corrosion of steel sheet piling. Corrosion Science 79 (2014) 139-147.

18. GB/T 4336-2016, Carbon and low-alloy steel-Determination of multi-element contents-Spark discharge atomic emission spectrometric method(routine method), 2016.

19. JB/T 7901-2001, Metals materials-Uniform corrosion-Methods of laboratory immersion testing, 2001.

20. GB/T 4336-2016, Carbon and low-alloy steel-Determination of multi-element contents-Spark discharge atomic emission spectrometric method(routine method), 2016.

21. NACE RP 0775-2005, Preparation, installation, analysis and interpretation of corrosion coupons in oilfield operations, 2005.

22. Qingli Cheng, Bin Tao, Liying Song, et al. Corrosion behaviour of Q235B carbon steel in sediment water fromcrude oil. Corrosion Science 111 (2016) 61-71.

23. Y.Y. Li, Z.Z. Wang, X.P. Guo, et al. Galvanic corrosion between N80 carbon steel and 13Cr stainless steel under supercritical CO2 conditions. Corrosion Science 147 (2019) 260-272.

24. Emmanuel Rocca, Hadri Faiz, Philippe Dillmann, et al. Electrochemical behavior of thick rust layers on steel artefact: Mechanism of corrosion inhibition. Electrochimica Acta 316 (2019) 219-227. 